\section{Concordância entre informações do Cartão da Gestante e da memória materna sobre assistência pré-natal}

\author{
Agreement between information from the \\ Pregnant Card and the mother's memory \\ of antenatal care
}

Edson Theodoro dos Santos Neto 1 Maria do Carmo Leal 2 Adauto Emmerich Oliveira 1 Eliana Zandonade 3 Silvana Granado Nogueira da Gama ${ }^{2}$

\footnotetext{
${ }^{1}$ Departamento de Medicina Social, Universidade Federal do Espírito Santo, Vitória, Brasil.

2 Escola Nacional de Saúde Pública Sergio Arouca, Fundação Oswaldo Cruz, Rio de Janeiro, Brasil. 3 Programa de Pós-graduação em Saúde Coletiva,

Universidade Federal do Espírito Santo, Vitória, Brasil.

Correspondência E. T. Santos Neto Departamento de Medicina Social, Universidade Federal do Espírito Santo.

Av. Marechal Campos 1468, Vitória, ES 29040-090, Brasil. edsontheodoro@uol.com.br
}

\begin{abstract}
This cross-sectional study aimed to verify agreement between information given by mothers after delivery and data recorded on Pregnant Cards about antenatal care under the Brazilian Unified National Health System in the Metropolitan Region of Vitória, Espírito Santo State, Brazil. The study considered a population of 1,035 postpartum mothers interviewed in eight hospitals, where the cards were copied. The representativeness of the sample was guaranteedby stratification according to the proportion of births. Kappa and McNemar tests were carried out with the collected and processed information. Agreement levels regarding antenatal care were predominantly poor (kappa < 0.20). Mothers tend to: overestimate the number of antenatal visits (McNemar = 51.73; p-value =0.001); affirm diseases during pregnancy, such as diabetes, anemia, hypertension and urinary infections; report the performance of laboratory tests; report the carrying out of clinical examinations. Results suggest the need to reflect on the type of data used for planning and implementing maternal and child public health polices, since data varies depending on the information source.
\end{abstract}

Prenatal Care; Health Evaluation; Bias (Epidemiology)

\section{Introdução}

A assistência pré-natal tem sido foco de investigação no Brasil 1 e no mundo 2 . Desde a medicalização do parto no século XVIII, as políticas relacionadas à saúde materno-infantil vieram se intensificando para abranger desfechos positivos em saúde, desde a concepção até a gestação e o puerpério ${ }^{3}$, que são fenômenos fisiológicos naturalmente esperados no desenvolvimento da espécie humana, mas sujeitos a intercorrências desfavoráveis.

Por isso, estudos epidemiológicos permitem identificar que o cuidado com a saúde e possíveis intervenções médicas, nesses momentos específicos do ciclo da vida humana, podem minimizar muito o risco de complicações e óbitos do recémnascido e da parturiente, além da redução de fatores de risco de mortalidade, como o baixo peso ao nascer e prematuridade 1 . Evidências revelam que em países de média e baixa renda o número reduzido de consultas de acompanhamento prénatal é fator de risco significante para aumento da mortalidade perinatal ${ }^{4}$. Além disso, o tempo de permanência de bebês em unidade intensivas de tratamento é muito maior quando a assistência pré-natal não é adequada 5 .

Contudo, um acompanhamento eficaz de pré-natal vai muito além da quantidade de encontros que a gestante tem com profissionais de saúde. A Organização Mundial da Saúde (OMS) avalia que um pré-natal realizado com núme- 
ro reduzido de consultas bem conduzidas, em gestações de baixo risco, pode ser tão efetivo quanto a realização de várias consultas ${ }^{6}$. No que se refere aos cuidados primordiais, evidências revelam que o controle e tratamento de infecções urinárias, HIV, sífilis, malária, verminoses e doença periodontal durante gestação podem reduzir o risco de óbito fetal 7 . Além disso, o diagnóstico precoce, monitoramento e tratamento da hipertensão arterial e do diabetes mellitus, somados ao acompanhamento dos sinais fetais, suplementação alimentar com ácido fólico, ferro, vitamina A, cálcio e magnésio, são evidências de que cuidados médicos podem reduzir o risco da mortalidade perinatal 7,8,9.

O Ministério da Saúde regulamentou no ano 2000 as principais atividades a serem desenvolvidas durante qualquer acompanhamento de prénatal em todo Brasil, incluindo seu início até o 4o mês, realização de seis ou mais consultas, de exames de tipagem sanguínea, de fator Rh, de VDRL, de dosagem de hemoglobina e hematócrito, de glicemia do jejum, de testagem anti-HIV, além da administração da vacina antitetânica, classificação do risco gestacional e realização de atividades educativas 10 . O estabelecimento de tais parâmetros foi fundamental para estabelecer critérios claros de avaliação nos serviços de saúde pública, principalmente no que se refere à assistência pré-natal na atenção básica articulada a média e alta complexidade, tanto ambulatorial quanto hospitalar, visando oferecer uma atenção humanizada à mulher e à criança 11 .

Por conseguinte, diversos estudos epidemiológicos avaliativos sobre assistência pré-natal têm sido conduzidos no Brasil para vislumbrar a qualidade do acompanhamento pré-natal, utilizando-se critérios consagrados internacionalmente, como os indicadores de Kessner ${ }^{1}$ e Kotelchuk 12 ou propostas nacionais 13,14 . No entanto, os métodos para obtenção das informações têm variado entre a auditoria de cartões de gestante 13 , entrevista com puérperas 12 , análise de prontuário de acompanhamento gestacional 14 ou a combinação de fontes 15 .

Supondo que informações sobre acompanhamento pré-natal podem diferir de acordo com a fonte de informação utilizada, este estudo propõe verificar a concordância entre as informações prestadas por puérperas e as registradas nos cartões das gestantes sobre assistência prénatal no Sistema Único de Saúde (SUS) da Região Metropolitana da Grande Vitória, Espírito Santo, Brasil.

\section{Métodos}

Um estudo epidemiológico seccional foi desenvolvido na Região Metropolitana da Grande Vitória, que abrigam quase metade da população total do Estado do Espírito Santo e cerca de sessenta por cento da população urbana (Instituto de Apoio à Pesquisa e ao Desenvolvimento Jones dos Santos Neves. http://www.ijsn.es.gov.br/, acessado em 09/Jul/2007). Nesses municípios, situam-se oito principais maternidades públicas e conveniadas ao SUS que assistem às puérperas da região produzindo grande volume de partos.

O universo amostral foi composto por todas as mulheres que se internaram em maternidades públicas ou conveniadas ao SUS localizadas na Região Metropolitana da Grande Vitória, por ocasião do parto, no período de abril a setembro de 2010. A amostra foi definida pela fórmula de tamanho amostral para estimar a proporção de nascidos vivos cobertos por sete ou mais consultas de pré-natal, considerando a população de 17.980 nascidos vivos em 2007, proporção esperada igual a $58,2 \%$, correspondente ao município com pior indicador de cobertura, ambos de acordo com o Sistema de Informações sobre Nascidos Vivos (SINASC). A precisão desejada foi de $4 \%$, o efeito do desenho igual a 1,5 e o nível de significância de 5\%. Esses cálculos resultaram num tamanho amostral de 849 mulheres. O total foi aumentado em cerca de $30 \%$ para considerar as possíveis perdas ou recusas, o que resultou em 1.131 abordagens e uma população de estudo de 1.035 puérperas.

Considerando as diferenças de contingente populacional de nascidos vivos entre os municípios, a representatividade da amostra foi garantida obedecendo à estratificação de acordo com as seguintes proporções: Cariacica (22,6\%), Fundão (1\%), Guarapari (6,3\%), Serra (26,3\%), Viana $(3,7 \%)$, Vila Velha $(22,2 \%)$ e Vitória $(17,9 \%)$.

Sete entrevistadoras de campo foram selecionadas, após a aprovação em testes teóricos e práticos do curso de treinamento de entrevistadores, promovido por professores da Universidade Federal do Espírito Santo (UFES) e Escola Nacional de Saúde Pública, Fundação Oswaldo Cruz (ENSP/Fiocruz). Além disso, um estudo piloto foi conduzido com 67 puérperas - não incluídas no estudo principal - para aprimoramento do formulário de pesquisa e treinamento das entrevistadoras.

Essas entrevistadoras visitavam no mínimo uma vez por semana todas as oito maternidades incluídas no estudo, abordavam mulheres no momento após o parto, para relato de informações sobre a assistência pré-natal. Nos dias das visitas, as entrevistadoras listavam em or- 
dem aleatória todas as puérperas residentes em um dos municípios da Região Metropolitana da Grande Vitória, em seguida, realizavam a amostragem sistemática intercalando-se de duas em duas para selecionar puérperas a serem abordadas. Esse método foi realizado até atingir o total da amostra.

$\mathrm{Na}$ abordagem, verificava-se a possibilidade da realização da entrevista e posse do Cartão da Gestante, excluindo-se mulheres que realizaram acompanhamento pré-natal (todo ou parte) no sistema privado ou em outros municípios fora da Região Metropolitana da Grande Vitória, puérperas com menos de 12 horas pós-parto cesáreo.

Após a identificação das puérperas sorteadas, as entrevistadoras explicavam os objetivos da pesquisa e solicitavam a assinatura do Termo de Consentimento Livre e Esclarecido, segundo as recomendações aprovadas pelo Comitê de Ética em Pesquisa do Centro de Ciências da Saúde da UFES, em 04 de novembro de 2009, sob protocolo no. 93/2009. Informações do prontuário médico da mulher e da criança foram transcritas no formulário, o cartão da gestante foi copiado na íntegra e, por último, a puérpera foi entrevistada. As variáveis do estudo foram construídas considerando as três fontes de informação para aumentar a completude das informações.

Perguntou-se à puérpera, sobre a realização de acompanhamento pré-natal, quantidade de consultas, mês gestacional da primeira e da última consulta, doenças pré-gestacionais e gestacionais, como diabetes mellitus, hipertensão, anemia e infecção urinária. Além disso, foi questionado sobre a administração de medicamentos e vacina antitetânica e a realização de exames de rotina do acompanhamento pré-natal.

De acordo com as informações colhidas no cartão da gestante, a quantidade de consultas de acompanhamento pré-natal foi obtida pela contagem dos registros. As únicas informações retiradas dos prontuários das puérperas foram a idade gestacional (método Capurro) e a data de nascimento da criança. De posse destas informações, foi calculada a idade gestacional na primeira e na última consulta. Informações sobre hipertensão arterial e diabetes mellitus pré-gestacionais foram retiradas do campo "antecedentes pessoais” no cartão da gestante. Considerouse que a gestante era portadora de hipertensão arterial quando a média da pressão arterial sistólica foi $\geq 140 \mathrm{mmHg}$ e diastólica $\geq 90 \mathrm{mmHg}$. Esse cálculo considerou no mínimo dois registros e no máximo seis registros de pressão nos cartões para ambas as medidas. A suspeita de diabetes gestacional foi definida pelo registro de pelo menos um nível glicêmico $\geq 105 \mathrm{mg} / \mathrm{dL}$. Já a diabetes gestacional confirmada considerou-se quando pelo menos em dois exames o nível glicêmico foi $\geq 105 \mathrm{mg} / \mathrm{dL}$ ou em apenas um dos exames o nível foi $\geq 200 \mathrm{mg} / \mathrm{dL}$. A anemia gestacional foi definida com base no registro de um nível de hemoglobina $<11 \mathrm{~g} / \mathrm{dL}$. O histórico de infecção urinária na gestação foi constatado pelos registros positivos para exames de urocultura. A administração de medicamentos e vacina antitetânica foi positiva quando anotada em qualquer local do cartão. A realização dos exames de tipagem sanguínea e fator Rh (ABO-Rh), VDRL, glicemia de jejum, exame de urina (EAS), dosagem de hemoglobina e hematócrito, testagem de HIV/AIDS e toque vaginal, além da repetição do VDRL, glicemia de jejum e EAS, foram considerados quando registrados no cartão.

A verificação dos batimentos cardíacos fetais foi considerada quando pelo menos um registro foi detectado nas três primeiras consultas. A pesagem e a medida da pressão arterial nas consultas de acompanhamento pré-natal foram calculadas pela razão entre o número de registros e o número de consultas anotadas. Em seguida, os resultados dos cálculos foram discriminados em categorias, segundo intervalos numéricos: nunca $(<0,40)$, às vezes $(0,40-0,79)$ e sempre $(>0,80)$.

O banco de dados foi construído a partir das informações digitadas no software SPSS versão 12.0 (SPSS Inc., Chicago, Estados Unidos). Antes da digitação, os formulários de pesquisa foram revisados por pesquisador para análise da completitude e consistência de informações, dando retorno aos entrevistadores de campo, em seguida, digitados por profissional estatístico. Após a digitação, o mesmo pesquisador realizava a conferência do banco de dados com os formulários. Por último, o pesquisador e o estatístico juntos realizaram uma última revisão conferindo todas as variáveis dos formulários com os dados do banco.

Em seguida, foram aplicados os testes de kappa, para mensurar os níveis de concordância, considerando segundo Landis \& Koch 16: concordância quase perfeita $(0,80-1,00)$, substancial $(0,60-0,79)$, moderada $(0,41-0,59)$, razoável $(0,21$ $0,40)$, ruim $(\leq 0,20)$. Além disso, foram aplicados os testes de kappa ajustado pela prevalência e de McNemar no programa PEPI versão 4.0 (Computer Programs for Epidemiologists; http://www. sagebrushpress.com/pepi), para verificar a direção (tendência) da discordância, adotando-se nível de significância estatística menor que 5\%. 


\section{Resultados}

A partir da amostra inicial de 1.035 puérperas entrevistadas, 23 (2,2\%) não obtiveram qualquer assistência em saúde durante a gestação e $6(0,6 \%)$ afirmaram ter realizado o acompanhamento prénatal, mas não portavam o cartão da gestante. Isso resultou o total de 1.006 cartões avaliados. A ausência de informações para algumas variáveis nos cartões e nos formulários da puérperas justificam a variação nos totais.

A Tabela 1 apresenta a distribuição dos dados faltantes para cada variável segundo as fontes de informação. Nota-se que as variáveis diabetes gestacional (confirmado), uso de medicamentos na gestação e vacinação antitetânica, foram as que apresentaram um percentual de ausência nos cartões maior que 50\%. Enquanto as variáveis relacionadas aos registros dos exames apresentaram um percentual de ausência em torno de 3\%, justificado pela ausência do cartão.

Considerando as demais variáveis, relacionadas à realização de atividades no pré-natal, os campos em branco representaram a não realização do exame. No que se refere a não-respostas das puérperas, nota-se que os percentuais de

Tabela 1

Distribuição dos dados faltantes, segundo as fontes de informação: Cartão da Gestante e entrevistas com puérperas sobre assistência pré-natal. Região Metropolitana da Grande Vitória, Espírito Santo, Brasil, 2010.

\begin{tabular}{|c|c|c|c|c|c|c|}
\hline \multirow[t]{2}{*}{ Variáveis } & \multicolumn{2}{|c|}{ Cartão (n = 1.006) } & \multicolumn{2}{|c|}{ Puérpera $(n=1.035)$} & \multicolumn{2}{|c|}{ Total } \\
\hline & $\mathbf{n}$ & $\%$ & $\mathbf{n}$ & $\%$ & $\mathbf{n}$ & $\%$ \\
\hline \multicolumn{7}{|l|}{ Acesso ao pré-natal } \\
\hline Quantidade de consultas pré-natais & 34 & 3,4 & 63 & 6,1 & 99 & 9,6 \\
\hline Mês gestacional da primeira consulta & 98 & 9,7 & 39 & 3,8 & 114 & 11,0 \\
\hline Mês gestacional da última consulta & 139 & 13,8 & 28 & 2,7 & 144 & 13,9 \\
\hline \multicolumn{7}{|l|}{ Doenças } \\
\hline Hipertensão pré-gestacional & 447 & 44,4 & 26 & 2,5 & 449 & 43,4 \\
\hline Hipertensão gestacional & 101 & 10,0 & 23 & 2,2 & 101 & 9,8 \\
\hline Anemia gestacional & 330 & 32,8 & 26 & 2,5 & 332 & 32,1 \\
\hline Diabetes pré-gestacional & 438 & 43,5 & 25 & 2,4 & 439 & 42,4 \\
\hline Diabetes gestacional (suspeito) & 318 & 31,6 & 23 & 2,2 & 318 & 30,7 \\
\hline Diabetes gestacional (confirmado) & 711 & 70,7 & 23 & 2,2 & 711 & 68,7 \\
\hline Infecção urinária & 352 & 35,0 & 26 & 2,5 & 355 & 34,3 \\
\hline \multicolumn{7}{|l|}{ Medicamentos } \\
\hline Medicamentos na gestacão & 628 & 62,4 & 25 & 2,4 & 630 & 60,9 \\
\hline Vacina antitetânica & 521 & 51,8 & 35 & 3,4 & 526 & 50,8 \\
\hline \multicolumn{7}{|l|}{ Exames laboratoriais } \\
\hline Exame ABORh & 29 & 2,9 & 37 & 3,6 & 43 & 4,2 \\
\hline Exame VDRL & 29 & 2,9 & 47 & 4,5 & 53 & 5,1 \\
\hline Exame de urina (EAS) & 29 & 2,9 & 26 & 2,5 & 32 & 3,1 \\
\hline Exame glicemia de jejum & 29 & 2,9 & 27 & 2,6 & 33 & 3,2 \\
\hline Exame hemoglobina & 29 & 2,9 & 34 & 3,3 & 40 & 3,9 \\
\hline Exame hematócrito & 29 & 2,9 & 34 & 3,3 & 40 & 3,9 \\
\hline Testagem HIV/AIDS & 29 & 2,9 & 27 & 2,6 & 33 & 3,2 \\
\hline Repetição exame VDRL & 29 & 2,9 & 49 & 4,7 & 55 & 5,3 \\
\hline Repetição do EAS & 29 & 2,9 & 29 & 2,8 & 35 & 3,4 \\
\hline Repetição glicemia de jejum & 29 & 2,9 & 36 & 3,5 & 42 & 4,1 \\
\hline \multicolumn{7}{|l|}{ Exames clínicos } \\
\hline Verificação dos batimentos cardíacos fetais & 31 & 3,1 & 25 & 2,4 & 31 & 3,0 \\
\hline Exame de toque vaginal & 29 & 2,9 & 23 & 2,2 & 29 & 2,8 \\
\hline Pesagem nas consultas & 32 & 3,2 & 23 & 2,2 & 32 & 3,1 \\
\hline Medida da pressão arterial & 32 & 3,2 & 24 & 2,3 & 33 & 3,2 \\
\hline
\end{tabular}


dados faltantes foram inferiores a $10 \%$ em todas as variáveis, o que reflete o treinamento adequado da equipe de entrevistadores de campo.

A soma dos totais apresentou grande variabilidade de acordo com a natureza das variáveis, visto que variáveis dependentes de exames diagnósticos e do preenchimento correto no cartão foram as que apresentaram maiores percentuais de dados faltantes. Deve-se destacar que os valores do total não são cumulativos, porque a ausên- cia de informação no cartão pode ter coincidido com a ausência no formulário.

Os resultados dos testes de concordância entre cartões das gestantes e da memória da puérpera, para as variáveis avaliadas são apresentados na Tabela 2. Nela são demonstrados os valores absolutos e relativos das discordâncias do cartão em relação à puérpera e da puérpera em relação ao cartão para evidenciar sua tendência. Além disso, são apresentados os valores para os

Concordância entre as informações prestadas pelas puérperas e as registradas nos Cartões das Gestantes sobre o acompanhamento pré-natal. Região Metropolitana da Grande Vitória, Espírito Santo, Brasil, 2010.

\begin{tabular}{|c|c|c|c|c|c|c|c|c|c|c|}
\hline \multirow[t]{2}{*}{ Variáveis comparadas } & \multicolumn{4}{|c|}{ Discordantes } & \multirow[t]{2}{*}{ Kappa } & \multirow[t]{2}{*}{ IC95\% } & \multirow{2}{*}{$\begin{array}{c}\text { Kappa } \\
\text { ajustado }\end{array}$} & \multirow{2}{*}{$\begin{array}{l}\text { Valor } \\
\text { de p }\end{array}$} & \multirow[t]{2}{*}{ McNemar } & \multirow{2}{*}{$\begin{array}{l}\text { Valor } \\
\text { de p }\end{array}$} \\
\hline & $\begin{array}{l}\text { Cartão vs. } \\
\text { puérpera }\end{array}$ & $\%$ & $\begin{array}{l}\text { Puérpera vs. } \\
\text { cartão }\end{array}$ & $\%$ & & & & & & \\
\hline \multicolumn{11}{|l|}{ Acesso ao pré-natal } \\
\hline Quantidade de consultas & 45 & 4,8 & 132 & 14,1 & 0,68 & $0,63-0,72$ & 0,72 & 0,001 & 48,4 & 0,001 \\
\hline $\begin{array}{l}\text { Mês Gestacional da primeira } \\
\text { consulta }\end{array}$ & 474 & 51,5 & 154 & 16,7 & 0,17 & $0,14-0,21$ & 0,23 & 0,001 & 212,8 & 0,001 \\
\hline Mês gestacional da última consulta & 175 & 19,6 & 124 & 13,9 & 0,25 & $0,20-0,30$ & 0,56 & 0,001 & 70,9 & 0,001 \\
\hline \multicolumn{11}{|l|}{ Doenças } \\
\hline Hipertensão pré-gestacional & 19 & 3,2 & 5 & 0,9 & 0,52 & $0,35-0,69$ & 0,92 & 0,001 & 7,3 & 0,007 \\
\hline Hipertensão gestacional & 0 & 0,0 & 150 & 16,1 & 0,08 & $0,03-0,13$ & 0,68 & 0,001 & 148,0 & 0,001 \\
\hline Anemia gestacional & 49 & 7,0 & 116 & 16,5 & 0,41 & $0,34-0,48$ & 0,53 & 0,001 & 26,4 & 0,001 \\
\hline Diabetes pré-gestacional & 3 & 0,5 & 4 & 0,7 & 0,46 & $0,12-0,79$ & 0,98 & 0,001 & 0,0 & 1,000 \\
\hline Diabetes gestacional (suspeito) & 3 & 0,4 & 19 & 2,6 & 0,44 & $0,24-0,63$ & 0,94 & 0,001 & 10,2 & 0,001 \\
\hline Diabetes gestacional (confirmado) & 7 & 2,2 & 10 & 3,1 & 0,56 & $0,37-0,75$ & 0,90 & 0,001 & 0,2 & 0,629 \\
\hline Infecção urinária & 18 & 2,6 & 140 & 20,6 & 0,33 & $0,25-0,40$ & 0,54 & 0,001 & 92,7 & 0,001 \\
\hline \multicolumn{11}{|l|}{ Medicamentos } \\
\hline Medicamentos na gestacão & 12 & 3,0 & 115 & 28,4 & 0,19 & $0,10-0,27$ & 0,37 & 0,001 & 81,9 & 0,001 \\
\hline Vacina antitetânica & 45 & 8,8 & 74 & 14,5 & 0,27 & $0,17-0,37$ & 0,53 & 0,001 & 6,6 & 0,010 \\
\hline \multicolumn{11}{|l|}{ Exames Laboratoriais } \\
\hline Exame ABORh & 16 & 1,6 & 338 & 34,1 & 0,11 & $0,07-0,14$ & 0,29 & 0,001 & 291,1 & 0,001 \\
\hline Exame VDRL & 27 & 2,7 & 253 & 25,8 & 0,10 & $0,05-0,15$ & 0,43 & 0,001 & 180,8 & 0,001 \\
\hline Exame de urina (EAS) & 8 & 0,8 & 326 & 32,5 & 0,05 & $0,02-0,08$ & 0,33 & 0,001 & 300,9 & 0,001 \\
\hline Exame glicemia de jejum & 26 & 2,6 & 261 & 26,0 & 0,07 & $0,02-0,12$ & 0,43 & 0,001 & 190,8 & 0,001 \\
\hline Exame hemoglobina & 20 & 2,0 & 272 & 27,3 & 0,09 & $0,04-0,13$ & 0,41 & 0,001 & 215,8 & 0,001 \\
\hline Exame hematócrito & 18 & 1,8 & 330 & 33,2 & 0,07 & $0,03-0,11$ & 0,30 & 0,001 & 277,9 & 0,001 \\
\hline Testagem HIV/AIDS & 16 & 1,6 & 323 & 32,2 & 0,07 & $0,03-0,11$ & 0,32 & 0,001 & 276,2 & 0,001 \\
\hline Repetição exame VDRL & 16 & 1,6 & 380 & 38,8 & 0,29 & $0,25-0,33$ & 0,19 & 0,001 & 332,8 & 0,001 \\
\hline Repetição do EAS & 14 & 1,4 & 431 & 43,1 & 0,24 & $0,20-0,28$ & 0,11 & 0,001 & 388,9 & 0,001 \\
\hline Repetição glicemia de jejum & 22 & 2,2 & 381 & 38,4 & 0,28 & $0,24-0,32$ & 0,19 & 0,001 & 318,0 & 0,001 \\
\hline \multicolumn{11}{|l|}{ Exames clínicos } \\
\hline $\begin{array}{l}\text { Verificação dos batimentos } \\
\text { cardíacos fetais }\end{array}$ & 8 & 0,8 & 61 & 6,1 & 0,09 & $-0,01-0,18$ & 0,86 & 0,001 & 67,0 & 0,001 \\
\hline Exame de toque vaginal & 11 & 1,1 & 541 & 53,8 & 0,07 & $0,05-0,10$ & $-0,10$ & 0,001 & 507,0 & 0,001 \\
\hline Pesagem nas consultas & 8 & 0,8 & 91 & 9,1 & 0,11 & $0,03-0,20$ & 0,84 & 0,001 & 26,9 & 0,001 \\
\hline Medida da pressão arterial & 23 & 2,3 & 68 & 6,8 & 0,11 & $0,02-0,21$ & 0,86 & 0,001 & 24,4 & 0,001 \\
\hline
\end{tabular}


testes de kappa, com intervalos de 95\% de confiança (IC95\%), os testes de kappa ajustado pela prevalência, os testes de McNemar e os níveis de significância para as estatísticas de teste.

Comparando a quantidade de consultas prénatais informadas pelas puérperas e coletadas nos cartões de gestantes, nota-se uma tendência de superestimação do número de consultas pelas puérperas em relação aos cartões (McNemar = 51,7 ; valor de $\mathrm{p}=0,001$ ) embora seja observada uma concordância substancial entre as fontes de informação (kappa $=0,68 ; p=0,001$ ). Quanto ao início e ao término do acompanhamento pré-natal, observa-se que as puérperas tendem a informar um início mais precoce do pré-natal $(\mathrm{McNemar}=212,8$; valor de $\mathrm{p}=0,001)$, com $u m$ nível de concordância ruim em relação aos dados do prontuário (kappa $=0,17$; valor de $\mathrm{p}=0,001$ ). O mesmo acontece com a informação sobre o término do pré-natal, com tendência a informar o mês de realização da última consulta mais precoce do que o calculado pelos registros do prontuário e do cartão (McNemar = 70,9; valor de $\mathrm{p}=0,001$ ), contudo com um nível de concordância razoável (kappa $=0,25$; valor de $\mathrm{p}=0,001$ ).

Sobre os resultados dos principais procedimentos diagnósticos e terapêuticos realizados durante o acompanhamento pré-natal, nota-se concordância moderada para o diagnóstico da hipertensão pré-gestacional, da anemia gestacional, do diabetes pré-gestacional e do diabetes gestacional suspeito e confirmado; concordância razoável para a vacinação antitetânica, para diagnóstico da infecção urinária, para a repetição dos exames deVDRL, EAS e glicemia de jejum; e, concordância ruim para todas as demais variáveis.

Todos os testes apresentaram valores estatisticamente significantes ao nível inferior 5\%, exceto para a variável diabetes gestacional confirmado, em que o kappa apresentou nível moderado, enquanto a tendência na discordância medida pelo teste de McNemar não foi observada. Outra exceção se refere ao estado de hipertensão prégestacional em que se percebe uma tendência materna de negação, em todas as demais variáveis, sejam relacionados a exames diagnósticos, procedimentos terapêuticos ou mesmo quadros patológicos, verificam-se afirmações maternas positivas que não se confirmam pelo cartão da gestante.

No que se refere às informações sobre procedimentos rotineiros executados no acompanhamento pré-natal: a pesagem nas consultas e a medida da pressão arterial, nota-se que as puérperas tendem a superestimar a realização desses procedimentos no pré-natal. Considerando o elevado teor de subjetividade envolvido nessa variável, a maioria delas opina que tais procedimentos sempre ocorreram, enquanto no registro verifica-se que estes às vezes ou nunca foram realizados. Por conseguinte, nota-se uma concordância ruim e uma discordância estatisticamente significante, sendo a concordância entre as fontes de informação sobre a medida da pressão arterial significante ao nível de $5 \%$.

Ao comparar as análises de kappa e kappa ajustado pela prevalência observam-se diferenças muito importantes. Isso ocorre prioritariamente quando o valor da discordância é pequeno e o nível de concordância é baixo. As variáveis que apresentaram tal característica foram: verificação dos batimentos cardíacos fetais (kappa $=0,09$; kappa ajustado $=0,86)$, pesagem nas consultas (kappa $=0,11$; kappa ajustado = 0,84 ) e medida da pressão arterial (kappa $=0,11$; kappa ajustado $=0,86$ ), contudo, para variáveis quantidade de consultas pré-natais e mês gestacional da primeira consulta, as análises de kappa ajustado não diferem muito dos testes de kappa. A única variável que apresentou kappa ajustado negativo foi a realização de exame de toque nas consultas em que a concordância foi muito ruim, enquanto para as variáveis relacionadas à repetição de exames de VDRL, EAS e glicemia de jejum os níveis de concordância ajustados diminuíram.

\section{Discussão}

A verificação da concordância entre as fontes de informação sobre assistência pré-natal é fundamental para se estabelecer perspectivas avaliativas do serviço de acompanhamento a gestantes. Nos estudos de avaliação, o Prontuário da Atenção Básica 17,18,19,20,21,22, o Cartão da Gestante 13 e as entrevistas com mãe 14 , gestantes 23 e puérperas 12 , e a combinação das fontes 1,15,24 têm sido métodos utilizados para obtenção de informações. Entretanto, algumas dificuldades têm sido encontradas no que se refere à assistência integrada à gestação, parto e puerpério, no SUS.

O acompanhamento pré-natal é realizado prioritariamente por médicos ou enfermeiros da atenção básica que elaboram o prontuário rotineiro de consultas, exames e procedimentos terapêuticos 17. Contudo, por mais completo que esteja, geralmente o prontuário não acompanha a gestante até o hospital ou maternidade, onde outro profissional realiza o parto sem nunca antes ter avaliado a mulher. Essa desarticulação entre os níveis de atenção de referência e contrar-referência é abordada por Zampieri \& Erdmann 25. Devido à falta de informação dos profissionais que realizam o parto, dados indispensáveis sobre o feto e a mãe, que implicam riscos para ambos, 
podem ser negligenciados ou exames e procedimentos terapêuticos podem ser repetidos desnecessariamente 26 .

O registro adequado dos dados no cartão da gestante é valorizado pelo Programa de Humanização no Pré-natal e Nascimento (PHPN) como fonte de informações do pré-natal da mulher 26. Nesse contexto, o Cartão da Gestante parece ser o elemento de ligação entre o acompanhamento gestacional na atenção básica, média e alta complexidade. Nele é possível registrar as informações essenciais da gravidez que implicam riscos para puérpera e criança.

A guarda do cartão pode ser vulnerável a perdas ou extraviamento, no entanto, Darmont et al. 27 analisam o discurso da valorização do cuidado com a saúde e a noção de importância da prevenção de puérperas HIV positivas que é traduzido pela preservação do Cartão da Gestante. Nota-se que as mulheres percebem o cartão como uma garantia para a realização de uma atenção ao parto sem maiores intercorrências, por isso o presente estudo encontrou poucas mulheres que realizaram o pré-natal sem o cartão na maternidade.

Isso mostra que a parturiente leva o cartão ao local do parto, entendendo que as informações são importantes, cumpre o papel de referenciamento, que deveria ser responsabilidade do sistema de saúde. Vale destacar que na maioria das vezes o cartão é fornecido, entretanto as informações registradas parecem não ser compartilhadas com as gestantes, o que sugere as diferenças encontradas, além, é claro, do precário preenchimento do cartão.

Contudo, propostas mais avançadas sugerem a criação de um sistema de informações integrado que permita o levantamento pelas maternidades ou hospitais das informações descritas no acompanhamento pré-natal 28 . Isso favoreceria a integração dos níveis de complexidade no SUS para atenção materno-infantil e a integralidade das ações em saúde. Mas, deve-se considerar que a informatização não garante qualidade de informação. Independente de ser manual ou digital, o que é de extrema relevância é a boa qualidade do preenchimento de dados nos sistemas de informação. Enquanto essas propostas não entram em vigor, esforços para preencher corretamente o Cartão da Gestante e guardá-lo a salvo de intempéries parecem ser responsabilidades a serem compartilhadas entre usuárias do SUS e profissionais de saúde 25 .

Os resultados do presente estudo demonstraram que as puérperas tendem a relatar que realizaram uma quantidade de consultas maior do que a registrada no cartão, além de referirem início e término do pré-natal mais precoce que o calculado pela idade gestacional de nascimento do bebê. Essas constatações, além de concordarem com Delgado-Rodríguez et al. 29 implicam diretamente as categorias de avaliação da qualidade da assistência pré-natal dos índices de Kessner 1 e de Kotelchuck 12, que consideram o número de consultas e o mês gestacional da primeira consulta como critérios de qualidade da assistência.

Além disso, existem repercussões significativas para avaliação e planejamento nos serviços de saúde no Brasil, no que se refere ao SINASC, em que se destaca um aumento expressivo de recém-nascidos cobertos por sete ou mais consultas de pré-natal nos últimos anos 30 , que pode não ser real. Visto que o preenchimento da informação sobre o número de consultas realizadas no pré-natal é realizado em um estabelecimento de saúde ou cartório sem especificação da fonte de informação, que pode ser a mãe da criança, o prontuário, ou o próprio cartão de pré-natal.

Considerando as informações sobre as principais doenças anteriores à gravidez ou desenvolvidas durante a gestação, as puérperas tenderam a não assumir a hipertensão pré-gestacional e a afirmar o desenvolvimento de doenças gestacionais, como hipertensão arterial, anemia, diabetes e infecção urinária, sem que estas estivessem diagnosticadas nos cartões. Essa tendência só não foi verificada para as variáveis diabetes prégestacional e diabetes gestacional (confirmado). Deve-se ressaltar que, mesmo presentes nos cartões, as informações pregressas foram fornecidas pela mulher e anotadas pelo pré-natalista, assim como as informações da entrevista. O estudo de Delgado-Rodríguez et al. 29 também demonstrou um nível de concordância razoável ou moderada, entre o relato da hipertensão arterial e anemia gestacional das puérperas, e o registro do prontuário hospitalar de pré-natal. Contudo, para o diagnóstico do episódio de infecção geniturinária o estudo revelou uma concordância muito superior ao presente estudo, possivelmente devido à alta especificidade dos critérios e à valorização dos casos graves, em detrimento dos casos leves, limítrofes ou suspeitos, na relação entre profissional de saúde e usuário do sistema. Tais achados possuem influência direta em estudos que consideram o diagnóstico dessas doenças como fatores de risco para desfechos desfavoráveis maternos e infantis 4 .

Sobre o uso de remédios na gravidez, as puérperas tendem a afirmar uso de medicamentos não confirmados no cartão, o que resulta numa concordância ruim, próxima à concordância razoável encontrada pelos autores que estudaram viés de memória 29 , que justifica esse baixo nível pela ausência de registro das prescrições nos 
prontuários médicos de acompanhamento prénatal ou pela prática da automedicação. Considerando a vacinação antitetânica, que apresentou uma concordância razoável, em pouco mais de um terço (393) dos cartões a informação sobre a administração da dose de reforço ou primeira dose foi encontrada. O percentual 31,4\% foi descrito por Parada 21 em São Paulo e de $89,5 \%$ por Moura et al. 18 na Microrregião de Baturité no Ceará, ambos colhidos de prontuários da atenção básica. Já em auditoria em cartões de gestantes em Juiz de Fora, Minas Gerais, um percentual de $42,4 \%$ foi encontrado 13 o que demonstra uma variabilidade acentuada quanto ao registro da vacinação que é crucial para definir condutas no momento do parto.

Quanto à realização dos principais exames laboratoriais no pré-natal, as puérperas tendem a afirmar a execução de todos os exames preconizados pelo PHPN 10, o que ocasiona uma concordância ruim para todos os exames. Deve-se considerar que esse fenômeno pode ser também explicado pela falta de preenchimento das informações no cartão, tornando-se imprescindível a realização de estudos que testem a concordância entre prontuários, cartões e mulheres.

Já em relação à repetição dos exames de VDRL, EAS e glicemia de jejum, o nível de concordância aumenta, mas não chega a ser considerado moderado para nenhum deles. Coutinho et al. 13 destacam percentuais de registros superiores a $70 \%$ para registro dos exames pelo menos uma vez nos cartões, enquanto no presente estudo os percentuais variam de $58 \%$ para o exame do tipo e fator sanguíneo e $69 \%$ para a glicemia de jejum. Em oposição, enquanto o estudo por auditoria de cartões 13 registra um percentual em torno de $15 \%$ para a repetição dos exames de VDRL, glicemia e urina, neste estudo foram encontrados percentuais próximos a $30 \%$, semelhantes ao estudo de Parada 21.

Nesse contexto, pode-se supor que algumas mulheres conseguiram realizar os primeiros exames e repeti-los no SUS, mas um percentual aproximadamente equânime não conseguiu realizar nenhum único exame, concordando com as principais ideias/vivências descritas nos discursos analisados por Almeida \& Tanaka 31, que evidenciam como a organização do serviço e oferta de procedimentos influencia na realização dos exames. Supõe-se que dois elementos possam estar envolvidos nessa questão: se por um lado pode haver deficiências de organização dos serviços de saúde com ausência insuficiente de oferta de exames, por outro existe uma cobrança social de responsabilidade para que as gestantes assumam uma postura de autocuidado, fazendo com que estas tenham a sensação do “dever cumprido", como "boas mães" durante a gravidez, quando afirmam que realizaram exames sem sequer ter consciência de suas verdadeiras atitudes. Sobre essa questão Zampieri \& Erdmann 25 exemplificam o reforço da ideia de realização do acompanhamento pré-natal adequado pelos discursos das gestantes como uma questão de responsabilidade com a sua própria vida e com a do novo ser.

Em relação aos principais exames clínicos realizados rotineiramente nas consultas de acompanhamento pré-natal encontramos concordâncias muito ruins para os exames ginecológicos e para a verificação dos batimentos cardíacos fetais. As mulheres tendem a afirmar que profissionais de saúde que conduziram o pré-natal realizaram tais procedimentos, contudo não se verifica nos cartões, o que sugere a possibilidade de confusão pelas puérperas. Embora em cerca de $90 \%$ dos cartões tenham sido encontrados os registros dos batimentos cardíacos fetais, semelhante a Coutinho et al. 13 e Carvalho \& Novaes 24 , somente em $8 \%$ o exame de toque vaginal foi registrado, enquanto $53 \%$ das mulheres afirmaram terem sido submetidas ao exame, percentual em conformidade com estudo de Neumann et al. 14 . Além da negligência profissional quanto à anotação do exame clínico ginecológico no cartão, que explica a discordância entre memória materna e registro, o baixo percentual pode ser devido à desvalorização do exame clínico 22 .

Outros procedimentos de acompanhamento muito comuns são: a verificação do peso e a medida da pressão arterial, que neste estudo tiveram sua frequência superestimada pela puérperas, corroborando o estudo sobre a opinião, em que cerca de $98 \%$ das primigestas da cidade de Curitiba, Paraná, relataram que "sempre" esses procedimentos foram realizados durante o pré-natal 24. Os níveis de concordância tão ruins podem ser explicados pelo alto grau de subjetividade envolvida no entendimento e expressão das respostas maternas codificadas em categorias, contrastando com o alto nível de objetividade traduzido pelos cálculos baseados nos registros dos cartões. As mulheres tendem a expressar que esses procedimentos sempre foram executados, quando na verdade estes nunca ou às vezes foram registrados.

O método estatístico do ajuste de kappa pela prevalência permitiu aumentar em muitas vezes os níveis de concordância para a maioria das variáveis, entretanto para as variáveis repetição de exames e realização de toque vaginal, as concordâncias ajustadas foram menores. Isso revela a relevância da utilização do método para equilibrar possíveis distorções dos resultados. No entanto, deve-se ressaltar que isso não anula 
os resultados das demais análises, mas permite visualizar o fenômeno sobre diferentes pontos de vista.

\section{Conclusões}

Os níveis de concordância entre os dados encontrados nos Cartões das Gestantes e a memória materna, sobre a assistência pré-natal, foram predominantemente ruins. Destacando-se a superestimação da quantidade de consultas realizadas, além da tendência de negação materna da hipertensão pré-gestacional em oposição à afirmação de todas as demais doenças gestacionais: hipertensão, diabetes, anemia e infecção urinária. Adicionam-se a afirmação das puérperas sobre a realização e repetição de exames laboratoriais propostos no PHPN, que nunca foram registrados, e o relato sobre a realização de exames clínicos como a pesagem e a medida da pressão arterial com uma frequência aumentada em relação ao cartão.

Verifica-se que explicações de natureza diferenciadas estão envolvidas no contexto do estudo, onde se percebem que tanto falhas na interlocução entre profissional de saúde e usuário, quanto negligência profissional de registro de doenças e exames clínico-laboratoriais nos cartões das gestantes contribuem para níveis de concordância tão ruins. Além disso, investigações mais específicas contribuirão para explicitar a subjetividade envolvida na documentação do processo de assistência pré-natal.

Os resultados sugerem reflexão sobre as diferentes fontes de informação consultadas para elaboração de estudos epidemiológicos avaliativos, que geralmente são utilizados como ferramentas para planejamento e execuções de medidas de saúde pública no âmbito dos serviços de saúde voltados para assistência materno-infantil no SUS.

\section{Resumo}

Este estudo seccional objetivou verificar a concordância entre as informações prestadas por puérperas e as registradas nos cartões das gestantes sobre assistência pré-natal no Sistema Único de Saúde da Região Metropolitana da Grande Vitória, Espírito Santo, Brasil. Considerou-se uma população de estudo de 1.035 puérperas, entrevistadas em oito maternidades, onde os cartões foram copiados. A representatividade da amostra foi garantida pela estratificação segundo a proporção de nascidos vivos. Informações foram coletadas, processadas e submetidas aos testes de kappa e McNemar. Os níveis de concordância sobre assistência pré-natal foram predominantemente ruins (kappa < 0,20 ). Puérperas tendem a superestimar a quantidade de consultas pré-natais (McNemar $=51,73$; valor de $p=0,001$ ), afirmar doenças gestacionais, como diabetes, anemia, hipertensão e infecções urinárias, relatar a execução de exames laboratoriais e clínicos. Os resultados sugerem uma reflexão sobre dados utilizados para o planejamento de políticas em saúde pública materno-infantil, visto que há variação conforme a fonte de informação.

Cuidado Pré-natal; Avaliação em Saúde; Viés (Epidemiologia)

\section{Colaboradores}

E. T. Santos Neto participou do planejamento e concepção do estudo, coleta e análise dos dados, interpretação dos resultados e redação do manuscrito. M. C. Leal e S. G. N. Gama colaboraram no planejamento do estudo, interpretação dos resultados e redação do manuscrito. A. E. Oliveira contribuiu na concepção e planejamento do estudo, interpretação dos resultados e redação do manuscrito. E. Zandonade participou da concepção e planejamento do estudo, análise dos dados, interpretação dos resultados e redação do manuscrito.

\section{Agradecimentos}

À Fundação de Amparo à Pesquisa do Espírito Santo (FAPES) pelo financiamento. 


\section{Referências}

1. Wehby GL, Murray JC, Castilla EE, Lopez-Camelo JS, Ohsfeldt RL. Prenatal care effectiveness and utilization in Brazil. Health Policy Plan 2009; 24: 175-88.

2. Villar J, Ba'aqeel H, Piaggio G, Lumbiganon $\mathrm{P}$, Miguel Belizán J, Farnot U, et al. WHO antenatal care randomised trial for the evaluation of a new model of routine antenatal care. Lancet 2001; 357:1551-64.

3. Theodoro ESN, Alves KCG, Zorzal M, Lima RCD. Políticas de saúde materna no Brasil: os nexos com indicadores de saúde materno-infantil. Saúde Soc 2008; 17:107-19.

4. Dowswell T, Carroli G, Duley L, Gates S, Gülmezoglu AM, Khan-Neelofur D, et al. Alternative versus standard packages of antenatal care for low-risk pregnancy. Cochrane Database Syst Rev 2010; (10):CD000934.

5. Carroli G, Villar J, Piaggio G, Khan-Neelofur D, Gülmezoglu M, Mugford M, et al. WHO systematic review of randomised controlled trials of routine antenatal care. Lancet 2001; 357:1565-70.

6. World Health Organization. What is the effectiveness of antenatal care? (Supplement). Copenhagen: WHO Regional Office for Europe's Health Evidence Networks; 2005.

7. Menezes EV, Yakoob MY, Soomro T, Haws RA, Darmstadt GL, Bhutta ZA. Reducing stillbirths: prevention and management of medical disorders and infections during pregnancy. BMC Pregnancy Childbirth 2009; 9 Suppl 1:S4.

8. Haws RA, Yakoob MY, Soomro T, Menezes EV, Darmstadt GL, Bhutta ZA. Reducing stillbirths: screening and monitoring during pregnancy and labour. BMC Pregnancy Childbirth 2009; 9 Suppl 1:S5.

9. Yakoob MY, Menezes EV, Soomro T, Haws RA, Darmstadt GL, Bhutta ZA. Reducing stillbirths: behavioural and nutritional interventions before and during pregnancy. BMC Pregnancy Childbirth 2009; 9 Suppl 1:S3.

10. Brasil. Portaria ${ }^{\circ} .569$, de 1 de junho de 2000. Institui o Programa de Humanização no Pré-natal e Nascimento no âmbito do SUS. Diário Oficial da União 2000; 8 jun.

11. Área Técnica de Saúde da Mulher, Departamento de Ações Programáticas Estratégicas, Secretaria de Atenção à Saúde, Ministério da Saúde. Pré-natal e puerpério: atenção qualificada e humanizada - manual técnico. Brasília: Ministério da Saúde; 2006.

12. Leal MC, Gama SGN, Ratto KMN, Cunha CB. Uso do índice de Kotelchuck modificado na avaliação da assistência pré-natal e sua relação com as características maternas e o peso do recém-nascido no Município do Rio de Janeiro. Cad Saúde Pública 2004; 20 Suppl 1:S63-72.
13. Coutinho T, Teixeira MTB, Dain S, Sayd JD, Coutinho LM. Adequação do processo de assistência pré-natal entre as usuárias do Sistema Único de Saúde em Juiz de Fora-MG. Rev Bras Ginecol Obstet 2003; 25:717-24.

14. Neumann NA, Tanaka OU, Victora CG, Cesar JA. Qualidade e equidade da atenção ao pré-natal e ao parto em Criciúma, Santa Catarina, Sul do Brasil. Rev Bras Epidemiol 2003; 6:307-18.

15. Trevisan MR, De Lorenzi DRS, Araujo NM, Esber K. Perfil da assistência pré-natal entre usuárias do Sistema Único de Saúde em Caxias do Sul. Rev Bras Ginecol Obstet 2002; 24:293-9.

16 Landis JR, Koch GG. The measurement of observer agreement for categorical data. Biometrics 1977; 33:159-74.

17. Succi RCM, Figueiredo EN, Zanatta LC, Peixe MB, Rossi MB, Vianna LAC. Evaluation of prenatal care at basic health units in the city of São Paulo. Rev Latinoam Enferm 2008; 16:986-92.

18. Moura ERF, Holanda Jr. F, Rodrigues MSP. Avaliação da assistência pré-natal oferecida em uma microrregião de saúde do Ceará, Brasil. Cad Saúde Pública 2003; 19:1791-9.

19. Silveira DS, Santos IS, Costa JSD. Atenção pré-natal na rede básica: uma avaliação da estrutura e do processo. Cad Saúde Pública 2001; 17:131-9.

20. Nagahama EEI, Santiago SM. O cuidado pré-natal em hospital universitário: uma avaliação de processo. Cad Saúde Pública 2006; 22:173-9.

21. Parada CMGL. Avaliação da assistência pré-natal e puerperal desenvolvidas em região do interior do Estado de São Paulo em 2005. Rev Bras Saúde Matern Infant 2008; 8:113-24.

22. Gonçalves CV, Costa JSD, Duarte G, Marcolin AC, Lima LCV, Garlet G, et al. Avaliação da frequência de realização do exame físico das mamas, da colpocitologia cervical e da ultrassonografia obstétrica durante a assistência pré-natal: uma inversão de valores. Rev Assoc Med Bras 2009; 55:290-5.

23. Costa GD, Cotta RMM, Reis JR, Siqueira-Batista R, Gomes AP, Franceschini SCC. Avaliação do cuidado à saúde da gestante no contexto do Programa Saúde da Família. Ciênc Saúde Coletiva 2009; 14 Suppl 1:1347-57.

24. Carvalho DS, Novaes HMD. Avaliação da implantação de programa de atenção pré-natal no Município de Curitiba, Paraná, Brasil: estudo em coorte de primigestas. Cad Saúde Pública 2004; 20 Suppl 2:S220-30.

25. Zampieri MFM, Erdmann AL. Cuidado humanizado no pré-natal: um olhar para além das divergências e convergências. Rev Bras Saúde Matern Infant 2010; 10:359-67. 
26. Área Técnica Saúde da Mulher, Secretaria de Políticas de Saúde, Ministério da Saúde. Programa de Humanização no Pré-natal e Nascimento. Rev Bras Saúde Matern Infant 2002; 2:69-71.

27. Darmont MQR, Martins HS, Calvet GA, Deslandes SF, Menezes JA. Adesão ao pré-natal de mulheres HIV+ que não fizeram profilaxia da transmissão vertical: um estudo sócio-comportamental e de acesso ao sistema de saúde. Cad Saúde Pública 2010; 26:1788-96.

28. Secretaria de Estado da Saúde do Espírito Santo. Governo lança projeto Rede da Mulher e da Criança. http://www.saude.es.gov.br/default.asp (acessado em 24/Mar/2010).
29. Delgado-Rodríguez M, Gómez-Olmedo M, Bueno-Cavanillas A, García-Martín M, Gálvez-Vargas R. Recall bias in a case-control study of low birth weight. J Clin Epidemiol 1995; 48:1133-40.

30. Moraes MS, Kujumjian FG, Chiaravalloti NF, Lopes JCC. Avaliação da assistência às gestantes: o caso do município de São José do Rio Preto, São Paulo, Brasil. Rev Bras Saúde Matern Infant 2004; 4:37584.

31. Almeida CAL, Tanaka OY. Perspectiva das mulheres na avaliação do Programa de Humanização do Pré-Natal e Nascimento. Rev Saúde Pública 2009; 43:98-104.

Recebido em 08/Jul/2011

Versão final reapresentada em 22/Set/2011 Aprovado em 20/Out/2011 